\title{
Assessment of Possible Link of Intestinal Microbiota and Type 2 Diabetes Mellitus
}

\author{
George Gborienemi Simeon ${ }^{*}{ }^{\mathbb{D}}$, Arikekpar Ibemologi $^{1}$, Dienize George Cameron ${ }^{2}$ \\ ${ }^{1}$ Department of Medical Laboratory Science, Niger Delta University, Wilberforce Island, Yenagoa, Nigeria \\ ${ }^{2}$ Department of Biochemistry, University of Port Harcourt, Yenagoa, Nigeria \\ Email: *ozunugborie@gmail.com, ariko2000200@yahoo.com, zenbosiv@gmail.com
}

How to cite this paper: Simeon, G.G., Ibemologi, A. and Cameron, D.G. (2021) Assessment of Possible Link of Intestinal Microbiota and Type 2 Diabetes Mellitus. American Journal of Molecular Biology, 11, 63-72.

https://doi.org/10.4236/ajmb.2021.113006

Received: January 7, 2021

Accepted: June 15, 2021

Published: June 18, 2021

Copyright $\odot 2021$ by author(s) and Scientific Research Publishing Inc. This work is licensed under the Creative Commons Attribution International License (CC BY 4.0).

http://creativecommons.org/licenses/by/4.0/

\section{Open Access}

\begin{abstract}
Introduction: This study assessed the role of gut bacteria in the development of type 2 Diabetes Mellitus. Methodology: Using bacteria cultural method, microbial species were isolated from feacal materials, identified and quantitated through application of genomic spectrophotometric systems with a quantitation of some marker biochemical parameters for Diabetes. Result: We observed a concentration of firmicutes, bacteriodetes, protecbacteria and bifidobacterium with Escherichia coli population predominating. Biochemical parameters reveal a 3 -fold raised value for some bromakers in type 2 diabetes. At a confidence interval of $95 \%$ paired sample test results gave significant differences for all tested pairs. Conclusion: Result suggests that microbiomes have the potential to alter the gut environment and cause changes that promote the development of type 2 diabetes.
\end{abstract}

\section{Keywords}

Assessment, Link, Microbiota, Microbiomes, Type 2 Diabetes

\section{Introduction}

Our current understanding of the complications of Diabetes mellitus and the apparent difficulties in providing a cure has elicited great concern.

The few drugs currently used in management of the disease have not proved effective enough when viewed along the complications associated with it such as retinopathy, neuropathy, kidney dysfunction, reduced libido, foot ulcer and other metabolic derangements. To expand the vista of research to unearth this quagmire has become compelling considering the rate of morbidity and mortality.

In recent times, research endeavours in microbial biotechnology are beaming 
focus on studying individual genomes to studying microbial communities and their interactions in different ecosystems and ecological niche [1] [2].

Microbiome research has become extremely relevant as a result of system level approaches that encapsulate a high-throughput sequencing and a gamut of genomic, proteomics and bioinformatics technologies to elucidate the direction to follow.

Recently, it has been discovered that several human microbes have great implication for human health due to the fact that a great number of diseases can be linked to the gut microbiome by virtue of the enormous amount of genes they possess.

There is now a gleam of understanding that identifying the hub microbiota would target organisms responsible for microbes interactions and enhance target interventions as an innovation. Over the years, there has been rapid increase in type 2 Diabetes Mellitus constituting a widespread metabolic disorder. It is now known that our microflora is linked to obesity related type 2 Diabetes mellitus [3] [4]. Intestinal microbiota are known to be involved in the process of energy harvesting which accounts for the development of obesity and host immune development. Of particular importance is the role microbiota is known to play in the progress of prediabetes in which insulin resistance is involved.

Previous research [5] [6] have documented the fact that people with insulin resistance and altered composition of intestinal microbiota in obesity may change the intestinal permeability and thus increase metalbolic endotoxin secretion that can result in inflammation which are the precursors to the development of type 2 Diabetes. Bacteria species including bacteriodetes and eschericia coli are known to exert influence on the intestinal mucus and the glycocalyx layer inhibiting permeability. Moreover, changes in the gut of microbiota dependent bacteria cause changes in the gut-junction protein, endocannabinoid system and intestinal alkaline phosphatase [7]. It is now known that some probiotics can prevent the onset of diabetes by their action as interleukin (IL) IFN- $\gamma$ or even modulating anti inflammatory IL-10 production as elucidated in some studies [8]. Furthermore, other studies have revealed the suppression of serum glucose, insulin, leptin, c-peptide, glycated hemoglobin, GLP-level, inflammatory IL- 6 and TNF- $\alpha$ in adepose tissue, PPAR- $\gamma$ and Glut4 gene expression in high fructose-fed rats [9].

Studies have also shown that some probiotics such as yoghurt may also reduce hs-CRP concentration and improve HOMA-R score in gestation [10]. Moreover, assessment of lipid profile following probiotic use has resulted in reduction of serum total cholesterol, low density Lipoprotein both of which are incriminating factors in type 2 Diabetes mellitus. Of interest is the fact that microbiota and type 2 diabetes mellitus have been widely studied in diabetes retinopathy, artherosclerosis, cystic fibrosis, diabetic foot ulcer and alzheimer's disease [11] [12]. It is known that chronic calorie restriction or deprivation and intermittent fasting represent non-pharmacological strategies for prevention and treatment of dis- 
eases such as aging, obesity and diabetes.

Accumulating evidence from previous studies points to the fact that gut microbiota contribute significantly to unique biological functions such as energy metabolism, regulation of integrity of the gut, and metabolic signaling [13] [14].

A distinguishing example of an endogenous metabolite which has been converted to a beneficial secondary metabolite by microbiome is the production of secondary bile acids. Produced in the liver, they are highly hydrophobic and have the potential to cause colitis; however, intestinal bacteria are known to convert them to more hydrophilic, less toxic, secondary products of bile acids which inhibit nuclear receptors with a great physiologic function [15].

\section{Materials and Methods}

At the Federal Medical Centre and Niger Delta University Teaching Hospital Yenagoa, one hundred $(n=100)$ feacal and fasting blood samples were collected from known type 2 diabetes patients aged between 35 years to 45 years after obtaining their consent and having glucose concentration $>15.0 \mathrm{mmol} / \mathrm{L}$.

Another set of samples were collected from sixty patients $(n=60)$ who were non-diabetics based on their fasting blood glucose ranging from $3.5-6.0$ $\mathrm{mmol} / \mathrm{L}$.

Glucose concentration was determined biochemically by the glucose oxidase method (Randox kit, London), glycated haemoglobin (HbAIC) was quantitated by ion-exchange high performance liquid chromatography (HpLc-Esi/ms) approach utilizing uv detection. To determine the concentration of insulin CX9 Automated machine (Beckman) was used with Beckman Assay reagent for the automated analysis. The bound and free ligand were then separated using double antibody precipitation.

Inflammatory characteristics such as erythrocyte sedimentation Rate (ESR), white blood count (WBC) and C-Reactive Protein (CRP) were determined by Westergreen method, Naubear counting chamber and ELISA respectively.

To extract the bacterial DNA, approximately $3 \mathrm{ml}$ of the feacal sample was used by following standard protocol for ZR feacal DNA miniprep ${ }^{\mathrm{TM}}$ D6010 (Zymo Research, USA). Using UV-Visible spectropholometer (Nano-Drop, model 13,300, Thermo fisher scientific, USA), the concentration of the extracted DNA was determined. Samples with DNA concentration $>300 \mu \mathrm{g} / \mu \mathrm{l}$ or the equivalent of 60 $100 \mathrm{ng}$ were considered suitable and were further analyzed using bacterial $16 \mathrm{~s}$ primers probe and cloned plasmid as standard [16] [17]. Pearson chi-square test was used to compare quantitative parameters and two-way analysis of variance was used to identify changes in bacterial abundance with each group using bacterial specie and concentration count as factor.

\section{Result}

This study characterized some gut microbiota of 160 individuals who met the inclusion criteria for the study. Investigation was based on the taxonomic com- 
position of the feacal microbiomes base on phyum, class, order, genus, and species level. We identified firmicutes, bacteriodetes, protecbacteria and bifidobactenum. Our findings in the various subgroups are shown in Table 1 and Table 2.

Comparison of biochemical parameters of the type 2 diabetes and non-diabetes were made using characteristics considered as biomarkers of inflammation and diabetes progression such as Glucose concentration, insulin level, Glycated Haemoglobin HBAIc, ESR, C-Reactive Protein and white Blood count (Table 3).

Table 1. Species variables of selected microbiota in the study and their concentration.

\begin{tabular}{ccc}
\hline & Diabetics & Non-Diabetics \\
\hline Firmicutes & 2.8 & 2.3 \\
Staphylococcus & 3.4 & 3.0 \\
Enterococcus & 2.2 & 1.7 \\
Streptococcus & 3.0 & 2.4 \\
Feacal bacterium & & \\
Bacteriodetes & 1.8 & 1.5 \\
Bacteriodes & 2.0 & 1.3 \\
Parabacteriodes & 1.9 & 1.4 \\
Prevotella & & 1.5 \\
Protecbacteria & 1.7 & 2.1 \\
Helicobacter & 2.0 & 1.8 \\
Enterobacter & 2.8 & 2.0 \\
Klebsiella & 2.6 & \\
Haemophilus & & \\
\hline
\end{tabular}

Measured values represent $10^{9} / \mathrm{ml}$.

Table 2. Demonstrating relationship of optical density of standards to numerical equivalent of some of the various bacteria estimated by means of spectrophmetric method.

\begin{tabular}{cccc}
\hline \multicolumn{4}{c}{ Descriptive Statistics } \\
\hline N & Mean & Std. Deviation \\
\hline OPTICAL DENSITY & 10 & 5.5000 & 3.02765 \\
Staphylococus auerus & 10 & 2.0400 & 1.14232 \\
Streph pyrogen & 10 & 1.6800 & 1.14581 \\
Enterococcus & 10 & 4.5000 & 1.79134 \\
Escherichia coli & 10 & 5.0900 & 1.42318 \\
Salmonella & 10 & 3.6700 & 1.08120 \\
Klebsiellia & 10 & 3.0400 & 0.91676 \\
Helico bacter & 10 & 1.5200 & 1.19889 \\
Valid N (listwise) & 10 & & \\
\hline
\end{tabular}


Table 3. Characteristic glycemic and inflammatory parameters assessed for diabetics and non-diabetics.

\begin{tabular}{ccc}
\hline & Diabetics & Non-Diabetics \\
\hline Glucose $(\mathrm{mmol} / \mathrm{L})$ & $15.3 \pm 2.02$ & $4.5 \pm 1.20$ \\
Insulin $(\mu \mathrm{U} / \mathrm{ml})$ & $1.3 \pm 0.50$ & $4.3 \pm 2.22$ \\
HbAIc $(\mathrm{mmol} / \mathrm{L})$ & $13.5 \pm 3.21$ & $6.41 \pm 1.80$ \\
Triglyceride $(\mathrm{mmol} / \mathrm{L})$ & $2.34 \pm 0.70$ & $1.52 \pm 0.50$ \\
Total cholesterol $(\mathrm{mmol} / \mathrm{L})$ & $6.2 \pm 0.42$ & $5.2 \pm 0.81$ \\
Low density Lipoprotein LDL $(\mathrm{mmol} / \mathrm{L})$ & $4.3 \pm 0.25$ & $2.20 \pm 0.61$ \\
C-RP $(\mathrm{mg} / \mathrm{L})$ & $10.9 \pm 2.60$ & $6.20 \pm 0.9$ \\
ESR $(\mathrm{mm} / \mathrm{hr})$ & $68.3 \pm 4.00$ & $7.2 \pm 2.40$ \\
WBC $\left(\times 10^{9} \mathrm{cells} / \mathrm{L}\right)$ & $13.3 \pm 6.30$ & $5.3 \pm 2.140$ \\
\hline
\end{tabular}

Values are mean \pm SEM of triplicate measurements.

Statistical evaluation of biochemical parameters by comparison among diabetic and non-diabetes gave the following results. Glucose $0.8617 \pm 0.49777$, Insulin $0.05774 \pm 0.3333$, HbAic $0.36638 \pm 0.21153$, CRP $0.8663 \pm 0.5000$, ESR $2.04206 \pm 1.17898$, WBC $1.37961 \pm 0.79652$ standard deviation and standard error of mean respectively all significant at $\mathrm{P}<0.05$.

We observed significant differences in the concentration of parameters between cases and controls. Compared to control, cases had unfavourably high lipid, glycemic profile, glucose, with raised values for c-reactive protein, ESR and WBC.

\section{Discussion}

The understanding that microbiomes and microbiota population have a role to play in type 2 diabetes mellitus is a recent and emerging awareness. Diagnosis of diabetes has been on fasting, random, 2 hours post-prandial glucose values, glycated haemoglobin, insulin sensitivity test and clinical observations of polydipsia, polyphagia and polyuria. However, the complication of diabetes with concurrent effects on major organs of the body such as kidney, nephron, sex organs, neurons and high mortality and morbidity rate and associated co-morbidities dictates the need to expand the battery of investigation.

Medications for treatment of diabetes still remain a conjecture with several management options to ameliorate the dilapidating condition. This study sought to identify the hub microbiota to reveal target intervention as an innovation especially as in situ microbiome engineering is becoming a novel research frontier.

Observable changes in the microbiome, the microbial metabolome and their interaction with the body system are now known to be correlated with several illnesses. We identified such as those in the phyla firmicutes, bacteriodetes, protecbacteria and bifidobacterium. It is known that modified foods contributes to the variation in microbiomes [18] [19]. Using cultural methods, we isolated the bacteria and determined their concentration using spectrophometric methods 
(Table 1 and Figure 1) by measuring their turbidity coefficients.

The factor of diet as a motivating factor in lifestyle change has been studied [20]. Vegetable-rich food is known to possess a significantly high concentration of fibre degrading bacteria responsible for the synthesis of short-chain fatty acids (SCFA) and other metabolites beneficial to the human body [21]. It also supports the generation of host factors that modulate the response and expression of toll-like receptors, (TLR) Lectin Regenerating Islet derived protein Gamma (RegIII- $\gamma$ ) and several interleukins such as IL-8, IL-22 and IL-23. These immune boosters are said to prevent and alleviate the risk of acquiring a disease [22]. On the contrary, imported or westernized diets rich in animal protein, high fats abundance of harmful microbial metabolites such as indole derivatives, phenolic acid and Trimethylamine N-oxide (TMAO) which have the potential to change the environmental milieu of the gut and have caused increased inflammatory bowel disease (IBD) notably ulcerative colitis and Crohn's disease.

Over the years there has been consistent consumption of probiotics which have been quantitated to be greater than or equal to $10^{8} \mathrm{cfu}$ or organism per day. These comes in forms of medication prepared as chewing gums, lozenges, tablets, capsules or even as powders most of which have probiotics as their primary active ingredient or component. Studies have elucidated the fact that staphylococcus aureus produces superantigens that are capable of inhibiting the inflammatory process. This function is however negated by the fact that the production of biofilms affects the robust activation of immune system [23]. Signalling pathways that have been identified for Staphylococcus aureus includes Target of RNA III activating protein (TRAP), Nuclear Kappa Beta (NF-k $\beta$ ), Extracellular Signal Regulated Kinase (ERK) [24] [25] TLR-2, 3-Kinase/AKT, NF/KB, ERK and phosphotidylinositol [26]. Some other cohort studies have also identified the fact that Staphylococcus facilitates the production of ATP and has been incriminated in some superficial and deep skin infections notably cellutitis, erysipelas and impetigo and these are enhanced by its signaling pathway which is N.F. KB65 [27].

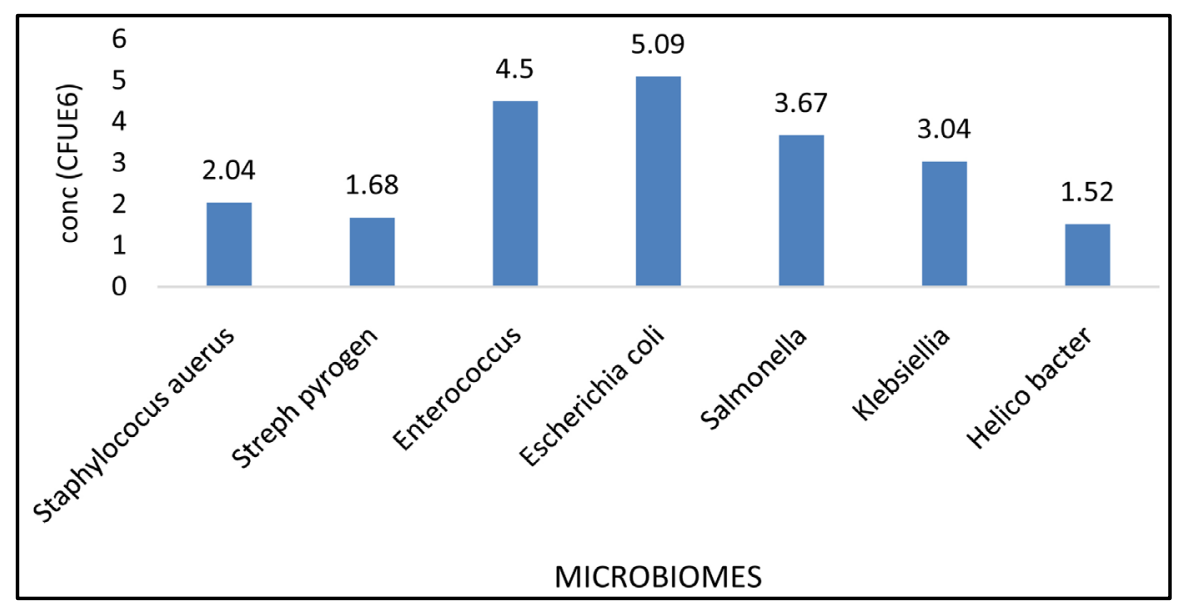

Figure 1. Graph showing concentration vs different bacterial species investigated. Values are representative of $10^{6} / \mathrm{ml}$, CFU colony forming units. 
The gut microbiota role in regulating metabolic process is also seen in its associated role in increased plasma Lipopolysaccharides, accumulation of triglyceride in the liver and inflammatory reaction. Raised level of triglyceride in this study is in tandem with some other previous works. Additionally, there was marked variation in the characteristics of parameters tested for the type $2 \mathrm{Di}$ abetes and controls.

Some earlier research studies have also shown how gut microbiota play significant role in progression of prediabetes condition which is related to insulin resistance.

Clinical studies have suggested that obese people with insulin resistance had altered composition of gut microbiota with attendant raised firmicutes/bacteriodetes ratio when compared with normal people [28]. The alteration in microbiota in obesity has been attributed to be responsible for the modulation of intestinal permeability, and elevated metabolic endotoxin secretion that enhances insulin resistance prompting the onset of type 2 diabetes Mellitus [29]. It has been reported [30] that Escherichia Coli and Bacteriodetes exhibited divergent behavior on the intestinal mucus and glycocalyx layer, a factor that can affect permeability.

Support for this has been given credence based on confirmation that some probiotics species have the capacity to regulate blood glucose homeostasis and improve type 2 diabetes mellitus by their action of down-regulating inflammatory IFN-Y, IL-2 and IL-IB and prompting IL-10 synthesis which plays anti-inflammatory role.

Some probiotic strains exhibit conducive autoxidative effect which favours the alleviation of pancreatic oxidative stress a possible cause of inflammation and apoptosis of the pancreatic B-cells [31].

Understanding how microbiomes are driving diseases states, leveraging on beneficial therapeutic bacterial microbiomes and development of biomarkers for disease diagnosis, patient stratification and personalized intervention were driving force for this research. The alignment of individual strain of bacteria with a particular functional activity, diseases pathway or mechanism will improve treatment outcome.

\section{Funding}

Funding for this project was provided by Geotech Medical Laboratory.

Equipment and reagents were provided by the Niger Delta University Molecular Biology Research Laboratory.

\section{Conflicts of Interest}

The authors declare no conflict of interest.

\section{References}

[1] Human Microbiome Project Consortium (2012) Structure, Function and Diversity 
of Healthy Human Microbiome. Nature, 486, 207-214.

https://doi.org/10.1038/nature11234

[2] Tilg, H. and Kaser, A. (2011) Gut Microbiome, Obesity and Metabolic Dysfunctions. Journals of Clinical Investigation, 12, 2126-2132.

https://doi.org/10.1172/JCI58109

[3] Langerberg, C. and Lott, L.A. (2018) Genomic in Sight into the Causes of Type 2 Diabetes. The Lancet, 391, 2463-2474. https://doi.org/10.1016/S0140-6736(18)31132-2

[4] Clarke, G. Stilling, R.M., Kennedy, P.J., Stanton, C., Cryan, J.F. and Dinan, T.G. (2014) Gut Microbiota: The Neglected Endocrine Organ. Molecular Endocrinology, 28, 1221-1238. https://doi.org/10.1210/me.2014-1108

[5] Devaraj, S., Hemarajatu, P. and Versalovic (2013) The Human Gut Microbiome and Body Metabolism Implications for Obesity and Diabetes. Clinical Chemistry, 59, 617-628. https://doi.org/10.1373/clinchem.2012.187617

[6] Lambert, S.M., Carson, T., Lowe, J., Ramarji, T., Leff, J.W. and Luo, L. (2015) Composition, Diversity and Abundance of Gut Microbiome in Prediabetes and Type 2 Diabetes. Journal of Diabetes and Obesity, 2, 1-7. https://doi.org/10.15436/2376-0949.15.031

[7] Claval, T., Desmarchelier, C. and Haller, D. (2014) Intestinal Microbiota in Metablic Diseases from Bacteria Community Structure and Function to Species of Pathophsiological Relevance. Gut Microbes, 5, 544-551. https://doi.org/10.4161/gmic.29331

[8] Kasatpibal, N., Whitney, J.D., Saokaew, S., Kenegkla, K., Heitkemper, M.M. and Apisanthan, A. (2017) Effectiveness of Probiotic, Prebiotic and Symbiotic Therapies in Reducing Post Operative Complications. A Systemic Review and Network Meta-Analysis. Clinical Infectious Diseases, 64, 153. https://doi.org/10.1093/cid/cix114

[9] Ye, J. (2013) Mechanisms of Insulin Resistance in Obesity. Frontiers in Medicine, 7, 14-24. https://doi.org/10.1007/s11684-013-0262-6

[10] Gareau, M.G., Sherman, P.M. and Walker, W.A. (2010) Probiotics and the Gut Microbiota in Intestinal Health and Disease. Nature, Reviews Gastroenterology and Hepatology, 7, 503-514. https://doi.org/10.1038/nrgastro.2010.117

[11] Beaney, A.J., Nunney, I., Gooday, C. and Dhatanya, K. (2016) Factors Determining the Risk of Diabetes Mellitus Foot Amputation. A Restrospective Analyst of a Tertiary Diabetes Foot Care Service. Diabetes Research and Clinical Practice, 114, 69-74. https://doi.org/10.1016/j.diabres.2016.02.001

[12] Tabur, S., Eren, M.A. and Celik, Y. (2015) The Major Predictors of Amputation and Length of Stay in Diabetes Patients with Acute Foot Ulceration. Wiener klinische Wochenschrift, 127, 45-50. https://doi.org/10.1007/s00508-014-0630-5

[13] Levy, M., Thaiss, C.A. and Zeevi, D. (2015) Microbiota Modulated Metabolites Shapes the Intestinal Microenvironment by Regulating NLRP6 Inflamasome Signaling. Cell, 163, 1428-1443. https://doi.org/10.1016/j.cell.2015.10.048

[14] Paharik, A.E. and Horswill, A.R. (2016) The Staphylococcal Biofilm: Adhesins, Regulation, and Host Response. Microbiology Spectrum, 4.

https://doi.org/10.1128/microbiolspec.VMBF-0022-2015

[15] Round, J.L. and Mazmanian, S.K. (2009) The Gut Nicrobiota Shapes Intestinal Immune Response during Health and Disease. Nature Reviews Immunology, 9, 313-323. https://doi.org/10.1038/nri2515

[16] Fadrosh, D.W., Na, B. and Giajer, P. (2014) An Improved Dual-Indexing Approach for Multiplexed 16srRNA Gene Sequencing on Illumina MiSeq Platform Microbi- 
ome. Microbiome, 2, 6. https://doi.org/10.1186/2049-2618-2-6

[17] Kuzynski, J., Stombaugh, J. and Walters, W.A. (2012) Using QIIME to Analyze 16s rRNA Gene Sequences from Microbial Communities. Current Protocols in Microbiology. https://doi.org/10.1002/9780471729259.mc01e05s27

[18] Ayei, F.A., Biagi, E. and Rampelli, S. (2018) Infant and Adult Gut Microbiome and Metabolome in Rural Bassa and Urban Settlers from Nigeria. Cell Reports, 23, 3056-3067. https://doi.org/10.1016/j.celrep.2018.05.018

[19] Riviere, A., Selak, M., Lantin, D., Leroy, F. and De Vuyst, L. (2016) Bifidobacteria and Butyrate-Producing Colon Bacteria; Importance and Strategies for Their Stimulation Human Gut. Frontiers in Microbiology, 7, 979.

https://doi.org/10.3389/fmicb.2016.00979

[20] David, L.A., Maurice, C.F., Carmody, R.N., Goolenberg, D.B., Button, J.E. and Wolfre, B.E. (2014) Diet Rapidly and Reproducibly Alters the Human Gut Microbiome. Nature, 505, 559-563. https://doi.org/10.1038/nature12820

[21] Everard, A. and Cani, P.D. (2013) Diabetes, Obesity and Gut Microbiota. Best Practices and Research Clinical Gastroenterology, 27, 73-83.

https://doi.org/10.1016/j.bpg.2013.03.007

[22] Lee, J. (2013) Adipose Tissue Microphages in the Development of Obesity Induce Inflammation, Insulin Resistance and Type 2 Diabetes. Archives of Pharmaceutical Research, 36, 208-222. https://doi.org/10.1007/s12272-013-0023-8

[23] De Angelis, M., Ferrocino, I. and Calabrese, F.M. (2020) Diet Influences the Functions of the Human Intestinal Microbiome. Scientific Reports, 10, Article No. 4247. https://doi.org/10.1038/s41598-020-61192-y

[24] Dominguez-Bello, M.G., Godoy-Victorino, F., Knight, R. and Blazer, M.J. (2019) Role of the Microbiome in Human Development. Gut, 68, 1108-1114. https://doi.org/10.1136/gutjnl-2018-317503

[25] Zhang, X., Shen, D., Fang, Z., Jie, Z., Qui, X. and Zhang, C. (2013) Human Gut Microbiota Changes Reveal the Progression of Glucose Intolerance. PLoS ONE, 8 , e71108. https://doi.org/10.1371/journal.pone.0071108

[26] Qui, J., Li, C., Zhu, J. and Zhang, F. (2012) A Metagenome-Wide Association Study of Gut Microbiota in Type 2 Diabetes. Nature, 490, 55-60. https://doi.org/10.1038/nature11450

[27] Perry, R.J., Peng, L., Barry, N.A., Cline, G.W., Zang, D. and Cardone, R.L. (2016) Acetate Mediates a Microbiome-Brain-B-Cell Axis to Promote Metabolic Syndrome. Nature, 534, 213-217. https://doi.org/10.1038/nature18309

[28] Brunkwall, L. and Orho-Melander, M. (2017) The Gut Microbiome as a Target for Prevention and Treatment of Hyperglycemia in Type 2 Diabetes from Current Human Evidence to Future Possibilities. Diabetologica, 60, 943-951. https://doi.org/10.1007/s00125-017-4278-3

[29] Larsen, N., Vogensen, F.K., Van den Berg, F.W., Nielsen, D.S., Andeasen, A.S. and Pedersen, B.K. (2010) Gut Microbiota in Human Adults with Type 2 Diabetes Differs from Non-Diabetic Adult. PLoS ONE, 5, e9085.

https://doi.org/10.1371/journal.pone.0009085

[30] Costea, P.I., Hildebrand, F.I., Arumugam, M., Backhed, F., Blaser, M.J. and Bushman, F.D. (2018) Enterotypes in the Landscape of Gut Microbial Community Composition. Nature Microbiology, 3, 8-16. https://doi.org/10.1038/s41564-017-0072-8

[31] De Filippo, C., Calvalieri, D., Di Paola, M., Ramazzotti, M., Poullet, J.B. and Massart, S. (2010) Impact of Diet in Shaping Gut Microbiota Revealed by Comparative 
Study in Children from Europe and Rural Africa. Proceedings of the National Academy of Sciences of the United States of America, 107, 14691-14696.

https://doi.org/10.1073/pnas.1005963107 